\title{
Robust motion control design for dual-axis motion platform using evolutionary algorithm
}

\author{
HORN-YONG JAN ${ }^{1}$, CHUN-LIANG LIN ${ }^{2 *}$, \\ CHING-HUEI HUANG ${ }^{2}$ and THONG-SHING HWANG ${ }^{1}$ \\ ${ }^{1}$ Graduate Institute of Electrical and Communications Engineering, Feng Chia \\ University, Taichung 40724, Taiwan, R.O.C. \\ $2 *$ Department of Electrical Engineering, National Chung Hsing University, \\ Taichung 402, Taiwan, R.O.C. \\ e-mail: chunlin@dragon.nchu.edu.tw
}

MS received 16 June 2007; revised 21 March 2008

\begin{abstract}
This paper presents a new approach to deal with the dual-axis control design problem for a mechatronic platform. The cross-coupling effect leading to contour errors is effectively resolved by incorporating a neural net-based decoupling compensator. Conditions for robust stability are derived to ensure the closedloop system stability with the decoupling compensator. An evolutionary algorithm possessing the universal solution seeking capability is proposed for finding the optimal connecting weights of the neural compensator and PID control gains for the $X$ and $Y$ axis control loops. Numerical studies and a real-world experiment for a watch cambered surface polishing platform have verified performance and applicability of our proposed design.
\end{abstract}

Keywords. Decoupling control; multi-objective optimization; evolutionary algorithm; platform; neural network.

\section{Introduction}

In the industrial applications, design for the single axis motion control systems has been well investigated with traditional or modern control strategies. Recently, precise contour control for the multi-axis systems has attracted much attention. As an example, a crosscoupling controller for coordination of two motor drivers proposed by Borenstein \& Koren (1985) was implemented. An application in robot control (Feng et al 1993) with the crosscoupling problem was discussed in which design and implementation of a cross-coupling motion controller was developed for minimization of the cross coupling error. Choi et al (2007) defined a position vector and applied a modified Hough transform to determine the dominant position error vector so as to correct the position error vector in a two-axis robot. To achieve a high degree of position and deposition accuracy, a coordination controller and

*For correspondence 
the use of pre-sliding friction characteristics were proposed to tracking control of $X-Y$ table (Park et al 2003 and Han \& Jafari 2007). $H_{\infty}$ control approaches were proposed by Kuo et al (2003) and Liu et al (2005) to deal with precise $X-Y-\theta$ motion control for a linear motor direct-drive $X-Y$ table. Although these methods were validated, selection of the weighting functions depends highly on the experience of robust control designs. The resulting high-order $H_{\infty}$ controllers were also hard to be implemented.

With regard to the intelligent control approaches, artificial neural networks (ANNs) have been widely applied to the mechatronic servo systems (Inshguro et al 1992) and visual control systems (Hashimoto et al 1992). Many attractive features of ANNs are praised such as ability of learning, function approximation, the mapping generation of input and output for unknown systems. These advantages render ANNs becoming a potential approach in control design for highly complicated plants.

Compared with the traditional analytical and optimization approaches, increasing advanced design methods resort to evolutionary algorithms (EAs) and genetic algorithms (GAs) for seeking the potential solutions. The algorithms copy the idea of survival of the fittest in natural selection, searching for the most suitable match type of species existence and try to reach the solution (Davis 1991). Recently, advanced control design methodologies have also been incorporated with these techniques to determine the optimal control gain for some specific applications, (Fang \& Xi 1997; Kim et al 1998; Kim et al 2001; Moallem et al 2001; Subbu et al 2005; Wai \& Tu 2007).

In this paper, a novel approach for decoupled dual-axis synchronous control is developed which combines an ANN compensator with two PID controllers determined via a modified multi-objective EV. The ANN serves as a decoupling compensator to improve the contour tracking accuracy and the PID controllers act to guarantee fundamental tracking performance for the $X$ and $Y$ axis control loops. Results about closed-loop stability are applied to the example and used as constraints for determining key parameters. Applicability of the approach has been numerically verified and experimentally applied to a watch cambered surface polishing system to conduct the planar and cambered surface polish.

\section{Structure of dual-axes system with cross-coupling control}

Throughout this paper, it should be noted that a linear operator $\mathfrak{I}$ which is not a function of $s$ denotes the operator in the time domain while $\Im(s)$ denotes its Laplace transform in the $s$ domain.

\subsection{Description of dual-axes system}

A dual-axis motion control system is considered. Its configuration, without cross-coupling, is illustrated in figure 1, in which $G_{p x}$ and $G_{p y}$ represent the models of the actuating devices for the $X$ and $Y$ axes, respectively, $G_{c x}$ and $G_{c y}$ are the respective controllers, $e_{x}$ and $e_{y}$ are, respectively, the position errors on the $X$ and $Y$ axes. In general, the error sources for a dual-axis system are classified into internal and external errors. The former is detected by the gears or ball-screw mechanisms. The latter makes evident when the gears or ball-screw mechanisms interact under different environmental conditions.

\subsection{Contour error}

Referring to figure 2, let $P_{d}=\left(P_{d x}, P_{d y}\right)$ denote the desired position for the moving platform's center, $P_{a}=\left(P_{a x}, P_{a y}\right) \triangleq(x, y)$ denotes the actual position and $P_{n}$ is the position on the 

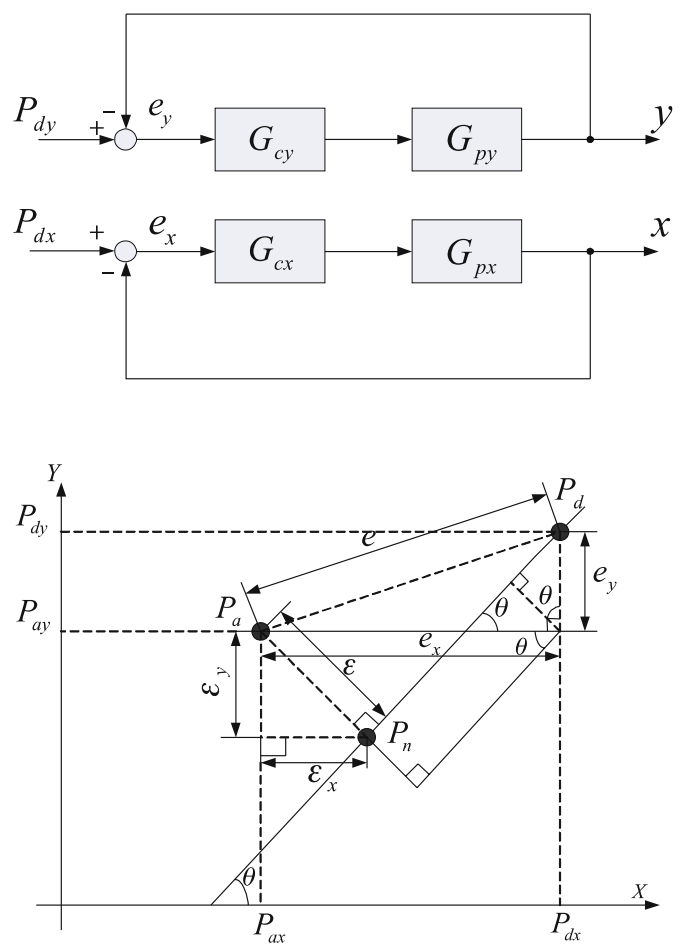

Figure 1. Dual axes motion control system without the cross-coupled effect.
Figure 2. Tracking and contour errors of the dual-axes motion control system.

reference trajectory that is closest to $P_{a}, e_{y}=P_{d y}-y$ and $e_{x}=P_{d x}-x$ are the tracking errors with respect to the corresponding axis.

The contour error is defined as

$$
\varepsilon=e_{x} C_{x}-e_{y} C_{y},
$$

where $\theta$ is the angle of the linear contour with respect to the $X$ axis, $C_{x} \triangleq \sin \theta$ and $C_{y} \triangleq \cos \theta$. Projections of the contour error on the $X$ and $Y$ axes are given by

$$
\varepsilon_{x}=e_{x} C_{x}^{2}-e_{y} C_{x} C_{y}, \quad \varepsilon_{y}=e_{x} C_{x} C_{y}-e_{y} C_{y}^{2} .
$$

Figure 3 illustrates configuration of the dual-axis cross-coupling system. The position control loop for each axis is equipped with a conventional PID controller. Extra compensation term,

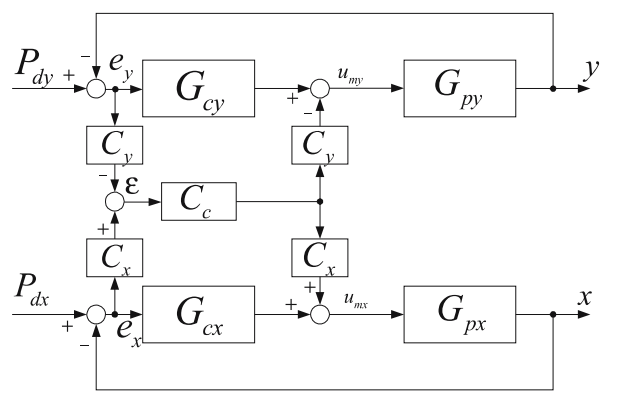

Figure 3. Configuration of the dual axes crosscoupling control system. 
denoted by $C_{c}$, is determined according to the tracking and contour errors with respect to the corresponding axis.

From figure 3, the output signals are

$$
x=G_{p x} u_{m x}, y=G_{p y} u_{m y},
$$

where $u_{m x}$ and $u_{m y}$ are the control commands and

$$
u_{m x}=G_{c x} e_{x}+C_{x} C_{c} \varepsilon, u_{m y}=G_{c y} e_{y}-C_{y} C_{c} \varepsilon,
$$

where $C_{c}$ is the decoupling controller. $G_{c x}$ and $G_{c y}$ are the conventional PID controllers defined by

$$
G_{c x}(s)=k_{p x}+k_{d x} s+k_{i x} \frac{1}{s}, G_{c y}(s)=k_{p y}+k_{d y} s+k_{i y} \frac{1}{s} .
$$

Combining Eqs. (2-4) gives

$$
x=G_{p x}\left[G_{c x}\left(P_{d x}-x\right)+C_{x} C_{c} \varepsilon\right], y=G_{p y}\left[G_{c y}\left(P_{d y}-y\right)-C_{y} C_{c} \varepsilon\right] .
$$

These give the governing equations for the whole system:

$$
\begin{aligned}
(1+ & \left.G_{p x} G_{c x}+G_{p x} C_{x} C_{c} C_{x}\right) x \\
& =G_{p x}\left(G_{c x}+C_{x} C_{c} C_{x}\right) P_{d x}-G_{p x} C_{x} C_{c} C_{y} P_{d y}+G_{p x} C_{x} C_{c} C_{y} y, \\
(1+ & \left.G_{p y} G_{c y}+G_{p y} C_{y} C_{c} C_{y}\right) y \\
& =-G_{p y} C_{y} C_{c} C_{x} P_{d x}+G_{p y}\left(G_{c y}+C_{y} C_{c} C_{y}\right) P_{d y}+G_{p y} C_{y} C_{c} C_{x} x .
\end{aligned}
$$

\subsection{Neural decoupling compensator}

Traditionally, the two controllers $G_{c x}$ and $G_{c y}$ are independently designed for the corresponding motion control system before considering the cross-coupling effect.

It is known that PID control designs based on the classical tuning techniques are not robust enough to accommodate variations of external disturbances, uncertain system parameters and structured perturbations. For the current system, there is also a contour error. A decoupling compensator control system based on an ANN is thus proposed.

The ANN acted as a decoupling compensator to compensate for the contour error induced by the cross coupling effect is an $L$-layered neural network denoted as $N N_{v}\left(v, W_{1}, W_{2}, \ldots, W_{L}\right)$, where $W_{i}(i=1, \ldots, L) \in R^{n_{i} \times n_{i-1}}$ are the weight matrices from the $(i-1)$ th layer to the $i$ th layer with the input $\varepsilon$. The neural decoupling compensator is expressed as

$$
C_{c}(\varepsilon)=\Psi_{L}\left[W_{L} \Psi_{L-1}\left[W_{L-1} \cdots \Psi_{2}\left[W_{2} \Psi_{1}\left[W_{1} \varepsilon\right]\right]\right]\right],
$$

where the nonlinear activation functions on the diagonal of the matrix operator $\Psi_{i}[\cdot]: R^{n_{i}} \mapsto$ $R^{n_{i}}$ operates component-wise on the activation value of each neuron and is defined as

$$
\Psi_{i}[\tau] \equiv \operatorname{diag}\left(\psi_{1}\left(\tau_{1}\right), \cdots, \psi_{n_{i}}\left(\tau_{n_{i}}\right)\right),
$$

where the activation functions associated with the hidden layers are

$$
F_{h} \equiv\left\{\psi(\cdot): R \mapsto R \mid \psi(\tau)=\lambda_{a c t}\left(\frac{1-e^{-\tau \beta}}{1+e^{-\tau \beta}}\right), \beta, \lambda_{a c t}>0\right\},
$$

where $\beta, \lambda_{\text {act }}$ and $\tau$ are used to adjust the shape of activation functions. The output layer is given as follows. 


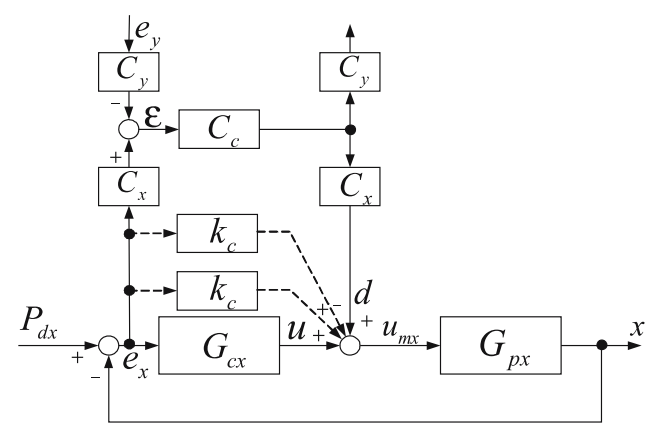

Figure 4. Single axis control system for stability analysis.

\section{Selection of the optimal parameters}

\subsection{Robust stability}

Stability of the inner system becomes a crucial issue here while incorporating the decoupling compensator into the system. This sub-section characterizes a quantitative condition in the sense of $L_{2}$-norm which ensures input-output stability of the whole closed-loop system with the PID controller and ANN.

We focus on the $X$ axis control system with the coupled perturbation as illustrated in figure 4 . The plant input is

$$
u_{m x}=u+d,
$$

where $u=G_{c x} e_{x}$ is the nominal control command and $d=f\left(e_{x}, e_{y}\right)$, where $f\left(e_{x}, e_{y}\right)=$ $C_{x} C_{c}\left(C_{x} e_{x}+C_{y} e_{y}\right)$.

Suppose that there is a gain $k_{c}$ so that $f\left(e_{x}, e_{y}\right)$ satisfies

$$
\left\|f\left(e_{x}, e_{y}\right)-k_{c} e_{x}\right\|_{2} \leq \gamma_{x}\left\|e_{x}\right\|_{2}+\gamma_{y}\left\|e_{y}\right\|_{2},
$$

where $\|f(t)\|_{2}=\sqrt{\int_{0}^{\infty} f^{2}(t) d t}$ denotes the $L_{2}$-norm and $0 \leq \gamma_{x, y}<\infty$. With this characterization, $e_{y}$ is treated as the bounded disturbance on the $X$ axis control system and the controller becomes

$$
\tilde{G}_{c x}(s)=\tilde{k}_{p x}+k_{d x} s+k_{i x} \frac{1}{s},
$$

where $\tilde{k}_{p x}=k_{p x}+k_{c}$. The system output is given by

$$
x=\left(1+G_{p x} \tilde{G}_{c x}\right)^{-1} G_{p x} \tilde{G}_{c x} P_{d x}+\left(1+G_{p x} \tilde{G}_{c x}\right)^{-1} G_{p x}\left[f\left(e_{x}, e_{y}\right)-k_{c} e_{x}\right] .
$$

For the nominal control design, $\tilde{G}_{c x}(s)$ should first ensure stability of the sub-systems $\frac{G_{p x}(s) \tilde{G}_{c x}(s)}{1+G_{p x}(s) \tilde{G}_{c x}(s)}$ and $\frac{G_{p x}(s)}{1+G_{p x}(s) \tilde{G}_{c x}(s)}$.

To proceed, the notion of truncated function $f(t)$ is introduced by

$$
f_{T}(t)=\left\{\begin{array}{ll}
f(t), & 0 \leq t \leq T \\
0, & t>T
\end{array} .\right.
$$


Then, taking norms and truncation with respect to both sides of Eq. (13) gives

$$
\begin{aligned}
& \left(1-\gamma_{x}\left\|\left(1+G_{p x} \tilde{C}_{c x}\right)^{-1} G_{p x}\right\|_{2}\right)\left\|x_{T}\right\|_{2} \\
& \leq\left(\left\|\left(1+G_{p x} \tilde{C}_{c x}\right)^{-1} G_{p x} \tilde{C}_{c x}\right\|_{2}+\gamma_{x}\left\|\left(1+G_{p x} \tilde{C}_{c x}\right)^{-1} G_{p x}\right\|_{2}\right)\left\|P_{d x T}\right\|_{2} \\
& \quad+\gamma_{y}\left\|\left(1+G_{p x} \tilde{C}_{c x}\right)^{-1} G_{p x}\right\|_{2}\left(\left\|P_{d y T}\right\|_{2}+\left\|y_{T}\right\|_{2}\right),
\end{aligned}
$$

where $0<T<\infty$ and $\left\|e_{y T}\right\|_{2} \leq\left\|P_{d y T}\right\|_{2}+\left\|y_{T}\right\|_{2}$.

Since $\frac{G_{p x}(s) \tilde{G}_{c x}(s)}{1+G_{p x}(s) \tilde{G}_{c x}(s)}$ is stable, by using the following relationship (Zhou \& Doyle 1998):

$$
\left\|\left(1+G_{p x} \tilde{C}_{c x}\right)^{-1} G_{p x} \tilde{C}_{c x}(t)\right\|_{2}=\left\|\frac{G_{p x}(j \omega) \tilde{C}_{c x}(j \omega)}{1+G_{p x}(j \omega) \tilde{C}_{c x}(j \omega)}\right\|_{\infty},
$$

where $\|G(j \omega)\|_{\infty}=\sup _{\omega} \bar{\sigma}[G(j \omega)]$ with $\bar{\sigma}(\cdot)$ denoting the maximum singular value, it is easy to see that if

$$
g_{1}=\gamma_{x}\left\|\frac{G_{p x}(j \omega)}{1+G_{p x}(j \omega) \tilde{G}_{c x}(j \omega)}\right\|_{\infty}<1
$$

then

$$
\left\|x_{T}\right\|_{2} \leq a_{1 x}\left\|P_{d x T}\right\|_{2}+a_{2 x}\left(\left\|P_{d y T}\right\|_{2}+\left\|y_{T}\right\|_{2}\right)
$$

where

$$
\begin{aligned}
& a_{1 x}=\frac{1}{1-g_{1}}\left(\left\|\frac{G_{p x}(j \omega) \tilde{C}_{c x}(j \omega)}{1+G_{p x}(j \omega) \tilde{G}_{c x}(j \omega)}\right\|_{\infty}+g_{1}\right)>0, \\
& a_{2 x}=\frac{\gamma_{y}}{1-g_{1}}\left\|\frac{G_{p x}(j \omega)}{1+G_{p x}(j \omega) \tilde{G}_{c x}(j \omega)}\right\|_{\infty}>0 .
\end{aligned}
$$

Clearly Eq. (15) constitutes a preliminary stability condition for the $X$ axis control system.

Similarly, one can obtain the stability condition for the $Y$ axis control system. If

$$
g_{2}=\gamma_{y}\left\|\frac{G_{p y}(j \omega)}{1+G_{p y}(j \omega) \tilde{G}_{c y}(j \omega)}\right\|_{\infty}<1
$$

then

$$
\left\|y_{T}\right\|_{2} \leq a_{1 y}\left\|P_{d y T}\right\|_{2}+a_{2 y}\left(\left\|P_{d x T}\right\|_{2}+\left\|x_{T}\right\|_{2}\right)
$$

where

$$
\begin{aligned}
& a_{1 y}=\frac{1}{1-g_{2}}\left(\left\|\frac{G_{p y}(j \omega) \tilde{C}_{c y}(j \omega)}{1+G_{p y}(j \omega) \tilde{G}_{c y}(j \omega)}\right\|_{\infty}+g_{2}\right)>0, \\
& a_{2 y}=\frac{\gamma_{x}}{1-g_{2}}\left\|\frac{G_{p y}(j \omega)}{1+G_{p y}(j \omega) \tilde{G}_{c y}(j \omega)}\right\|_{\infty}>0 .
\end{aligned}
$$


Combining Eqs. (16) and (18) gives

$$
\left[\begin{array}{cc}
1 & -a_{2 x} \\
-a_{2 y} & 1
\end{array}\right]\left[\begin{array}{c}
\left\|x_{T}\right\|_{2} \\
\left\|y_{T}\right\|_{2}
\end{array}\right] \leq\left[\begin{array}{ll}
a_{1 x} & a_{2 x} \\
a_{2 y} & a_{1 y}
\end{array}\right]\left[\begin{array}{c}
\left\|P_{d x T}\right\|_{2} \\
\left\|P_{d y T}\right\|_{2}
\end{array}\right] .
$$

Clearly, if

$$
g_{3}=a_{2 x} a_{2 y}<1
$$

then

$$
\left[\begin{array}{c}
\left\|x_{T}\right\|_{2} \\
\left\|y_{T}\right\|_{2}
\end{array}\right] \leq \frac{1}{1-a_{2 x} a_{2 y}}\left[\begin{array}{cc}
a_{1 x}+a_{2 x} a_{2 y} & a_{2 x}\left(1+a_{1 y}\right) \\
a_{2 y}\left(1+a_{1 x}\right) & a_{1 y}+a_{2 x} a_{2 y}
\end{array}\right]\left[\begin{array}{c}
\left\|P_{d x T}\right\|_{2} \\
\left\|P_{d y T}\right\|_{2}
\end{array}\right], \forall T \geq 0 .
$$

For $P_{d x}, P_{d y} \in L_{2}$ then

$$
\left[\begin{array}{l}
\left\|P_{d x T}\right\|_{2} \\
\left\|P_{d y T}\right\|_{2}
\end{array}\right] \leq\left[\begin{array}{l}
\left\|P_{d x}\right\|_{2} \\
\left\|P_{d y}\right\|_{2}
\end{array}\right], \forall T \geq 0 .
$$

This implies

$$
\left[\begin{array}{c}
\left\|x_{T}\right\|_{2} \\
\left\|y_{T}\right\|_{2}
\end{array}\right] \leq \frac{1}{1-a_{2 x} a_{2 y}}\left[\begin{array}{ll}
a_{1 x}+a_{2 x} a_{2 y} & a_{2 x}\left(1+a_{1 y}\right) \\
a_{2 y}\left(1+a_{1 x}\right) & a_{1 y}+a_{2 x} a_{2 y}
\end{array}\right]\left[\begin{array}{c}
\left\|P_{d x}\right\|_{2} \\
\left\|P_{d y}\right\|_{2}
\end{array}\right], \forall T \geq 0 .
$$

Since right-hand side of the above inequality is independent of $T$, it follows that $x, y \in L_{2}$.

The stability conditions derived in (15), (17) and (19) serve as strict constraints while determining the control gains and ANN's connecting weights. Selection of these parameters will be conducted using EA which is explained in detail in the following sections.

\subsection{Evolutionary algorithm}

EA is applied for seeking the feasible connecting weights of the neural network and six PID control gains from the permissible solution space. The idea of EA is to represent an individual as a pair of float-valued vector $v=\left(\underline{k}_{i}, N\left(0, \sigma^{2}\right)\right)$, where $\underline{k}_{i}$ represents a point in the search space and $N\left(0, \sigma^{2}\right)$ consists of independent random Gaussian numbers with zero mean and the standard deviation $\sigma$. The search starts by generating $\omega_{p}$ parents in each generation. Then, $\lambda=l \omega_{p}$ offsprings are generated by mutation, as a result of the addition of random numbers.

To utilize the depicted EA, the individual $\underline{k}_{i}$ of a generation, defined below, consists of six PID control gains and all connecting weights of the ANN:

$$
\underline{k}_{i}=\left[\underline{k}_{i, P I D}, \underline{k}_{i, N N}\right], i=1, \ldots, \omega_{p},
$$

where

$$
\begin{aligned}
\underline{k}_{i, P I D} & =\left[\underline{\tilde{k}}_{i, P x} \underline{k}_{i, I x} \underline{k}_{i, D x} \underline{\tilde{k}}_{i, P y} \underline{k}_{i, I y} \underline{k}_{i, D y}\right] \\
\underline{k}_{i, N N} & =\left[\operatorname{vec}^{T}\left(w_{i, j 1}^{i n}\right), \operatorname{vec}^{T}\left(w_{i, k j}^{(\mu+1)(\mu)}\right), \operatorname{vec}^{T}\left(w_{i, 1 k}^{\text {out }}\right), \operatorname{vec}^{T}\left(w_{i}^{\text {bias }}\right)\right], \\
j & =1, \cdots, j_{\mu}, k=1, \cdots, j_{\mu+1}, \mu=1, \cdots, L-1 .
\end{aligned}
$$


The mutants are generated by replacing the new individuals $\underline{k}_{i j}$ via

$$
\underline{k}_{i j}^{(g+1)}=\underline{k}_{i}^{(g)}+N\left(0, \sigma^{2}\right), i=1, \cdots, \omega_{p}, j=1, \cdots, l,
$$

where $j_{\mu}$ is the number of neurons per layer, $g$ is the index of the generation and $L$ is the number of hidden layers. Fixed standard deviations may encounter the situation of difficulty that the search cannot escape from the local solution. However, an appropriate variable standard deviation will speed up convergence of the solution search. For details regarding idea and operation of EAs, one could consult to Bäck (1996).

\subsection{Fitness function design}

Suitability of a valid parameter vector is determined by the fitness function denoted $O\left(\underline{k}_{i}\right)$. Combination of the neural network's connecting weights and PID control gains forms an individual of the EA. The optimal solution is to be determined via the EA-based optimization process with respect to the full operating range (in terms of the angle $\theta$ ) of the dual-axis control system. The following fitness function is defined:

$$
O\left(\underline{k}_{i}\right)=\exp ^{-\left(z_{0} C_{x y}+z_{1} E r r+z_{2} T r+z_{3} O s\right)},
$$

where $z_{0,1,2,3}$ are the weighting factors with respect to the performance indices; $E r r, T r$ and $O s$ are the normalized performance indices of steady-state error, rise time and maximum overshoot for the $X$ and $Y$ axis control loops; $C_{x y}$ denotes the cross-covariance between the coupled subsystems:

$$
C_{x y}=\frac{1}{t_{f}} \int_{0}^{t_{f}} x(t) y(t) d t-\frac{1}{t_{f}^{2}} \int_{0}^{t_{f}} x(t) d t \int_{0}^{t_{f}} y(t) d t,
$$

where $t_{f}$ is the total operating time.

The stability conditions derived in Eqs. (15), (17) and (19) are treated as the constraints while picking up the desired solution. A penalty function is introduced which converts the constrained optimization problem into an unconstrained one:

$$
S\left(\underline{k}_{i}\right)=1-\left[\frac{1}{3} \sum_{j=1}^{3} w_{c j} \frac{\Delta b_{j}}{\max \left\{\Delta b_{j}, \varepsilon_{s}\right\}}\right]^{\rho},
$$

where $\Delta b_{j}=\max \left\{0, g_{j}\left(\underline{k}_{i}\right)-1\right\}, w_{c j}$ is the weighting factor, $\rho$ is used to adjust the severity of the penalty functions and $\varepsilon_{s}$ is a tiny positive constant. The transformed fitness function is then modified as

$$
\bar{O}\left(\underline{k}_{i}\right)=O\left(\underline{k}_{i}\right) S\left(\underline{k}_{i}\right) .
$$

The process for the operation is summarized as follows.

(i) A population of $\omega_{p}$ parent solutions $\underline{k}_{i}, i=1, \cdots, \omega_{p}$ are initially randomly generated.

(ii) Each parent $\underline{k}_{i}$ creates $l$ offsprings $\underline{k}_{i j}^{\prime}$ by using

$$
\begin{aligned}
\underline{k}_{i j}^{\prime} & =\underline{k}_{i}+N\left(0, \sigma^{2}\right), \quad j=1, \ldots, l, \\
\sigma^{\prime} & =\sigma r^{\alpha}
\end{aligned}
$$


where the exponent $\alpha \triangleq N\left(0, \Delta \sigma^{2}\right)$ denotes the normal distribution with mean zero and variance $\sigma^{2}$ and $\Delta \sigma$ is the difference of $\sigma$ between the last two generations. The generated PID control gains should ensure stability of the nominal closed-loop sub-systems. Unqualified offsprings should be ignored and qualified one should be added.

(iii) Perform the closed-loop system simulation with step input. Each $\underline{k}_{i}^{\prime}$ is then scored in light of the constrained fitness function $\bar{O}\left(\underline{k}_{i}^{\prime}\right)$.

(iv) Each $\underline{k}_{i}^{\prime}$ competes against others. A 'winner' is assigned if its score is higher than its opponents.

(v) The $\omega$ solutions with the greatest number of wins are retained as the parents to the next generation.

The stopping criterion is adopted to terminate the search process when $\left|\bar{O}_{\max }^{(g)}-\bar{O}_{\min }^{(g)}\right|<\tilde{\xi}$ where $\bar{O}_{\max }=\max _{i=1, \ldots, \omega_{p}} \bar{O}_{i}$ and $\bar{O}_{\text {min }}=\min _{i=1, \ldots, \omega_{p}} \bar{O}_{i}$. Otherwise proceed to Step 2.

\section{Experiment and verification}

The platform under consideration is actuated by a linear brushless DC motor (LBDCM) and a rotary DC servo motor. For the unit step response, the system is required to have the rise time less than $1.2 \mathrm{sec}$, the settling time less than $2.0 \mathrm{sec}$ (use the $2 \%$ criterion), the maximum overshoot less than 5\% and zero steady state error. The driver with LBDCM and the rotary motor with a ballscrew are respectively modelled as

$$
G_{p x}(s)=\frac{171 \cdot 2}{s(s+13 \cdot 82)}(\mathrm{m} / \mathrm{V}), \quad G_{p y}(s)=\frac{266 \cdot 15}{s(s+12 \cdot 571)}(\mathrm{m} / \mathrm{V})
$$

For the decoupling compensator, the ANN is a 3-layered neural network. There are two neurons in the input layer, three neurons in the hidden layer and one neuron in the output layer. Including two PID controllers for the two drive loops there are totally 16 parameters to be determined. The parameters are selected through the application of EA with different $\theta$. The weighting factors $z_{j}$ of the fitness function are selected as $z_{0}=1 \cdot 5, z_{1}=0 \cdot 4, z_{2}=0 \cdot 2$ and $z_{3}=0.4$. For the penalty term, $\rho=3$ and $\varepsilon_{s}=0.01$. For the EA, $\omega_{p}=100, l=7$, $\tilde{\xi}=1 \times 10^{-5}$, and $1<r<5$. A series of simulations are conducted on Matlab to evaluate performance of the resulting control system.

\subsection{Case 1: Cross-coupled system without neural compensation}

For the cases of the angle $\theta$ from -90 to 90 degrees, the control gains alone converge after 25 evolutionary generations. The results are summarized in table 1 . Step responses of the two individual driving loops are shown in figure 5 while the angle $\theta$ is 15 degrees. It is seen that the prespecified specifications have been achieved; however, there is dissimilarity between the two subsystems because of the different electrical characteristics for the two motors. It proceeds to check the contour error when there is in the absence of the ANN decoupling compensator. Effectiveness of contour errors is displayed in figure 6. Clearly, without the decoupling compensator, the combined error of both $X$ and $Y$ axes will not be eliminated effectively. 
Table 1. Optimal PID control parameters with respect to the operating angle.

\begin{tabular}{|c|c|c|c|c|c|c|}
\hline \multirow{2}{*}{$\begin{array}{l}\text { Angle } \\
\text { (deg) }\end{array}$} & \multicolumn{3}{|c|}{ PID control gains for $x$ axis } & \multicolumn{3}{|c|}{ PID control gains for $y$ axis } \\
\hline & $k_{p x}$ & $k_{i x}$ & $k_{d x}$ & $k_{p y}$ & $k_{i y}$ & $k_{d y}$ \\
\hline $90^{\circ}$ & $2 \cdot 226 \mathrm{e}-3$ & $2 \cdot 286 \mathrm{e}-6$ & $9 \cdot 542 \mathrm{e}-4$ & $9 \cdot 454 \mathrm{e}-2$ & $6 \cdot 564 \mathrm{e}-4$ & $1.448 \mathrm{e}-3$ \\
\hline $75^{\circ}$ & $1 \cdot 910 \mathrm{e}-3$ & $2 \cdot 507 e-6$ & $3 \cdot 638 \mathrm{e}-4$ & $9 \cdot 785 \mathrm{e}-2$ & $1 \cdot 258 \mathrm{e}-3$ & $2 \cdot 221 \mathrm{e}-3$ \\
\hline $60^{\circ}$ & $2 \cdot 248 \mathrm{e}-3$ & $1.083 e-6$ & $9.792 \mathrm{e}-4$ & $9 \cdot 456 \mathrm{e}-2$ & $6 \cdot 563 e-4$ & $1.448 \mathrm{e}-3$ \\
\hline $45^{\circ}$ & $2 \cdot 173 e-3$ & $1 \cdot 122 \mathrm{e}-5$ & $7 \cdot 531 \mathrm{e}-4$ & $9.775 \mathrm{e}-2$ & $1 \cdot 256 \mathrm{e}-3$ & $3 \cdot 681 \mathrm{e}-4$ \\
\hline $30^{\circ}$ & $1.798 \mathrm{e}-3$ & $3.926 \mathrm{e}-6$ & $3 \cdot 066 \mathrm{e}-4$ & $9 \cdot 945 \mathrm{e}-2$ & $1 \cdot 189 \mathrm{e}-3$ & $9 \cdot 557 e-4$ \\
\hline $15^{\circ}$ & $2 \cdot 221 \mathrm{e}-3$ & $1 \cdot 808 \mathrm{e}-6$ & $8 \cdot 251 \mathrm{e}-4$ & $9 \cdot 025 \mathrm{e}-2$ & $1 \cdot 687 \mathrm{e}-5$ & $1.637 \mathrm{e}-3$ \\
\hline $0^{\circ}$ & $8 \cdot 679 \mathrm{e}-3$ & $4 \cdot 378 \mathrm{e}-5$ & $9 \cdot 867 \mathrm{e}-4$ & $9 \cdot 782 \mathrm{e}-2$ & $4 \cdot 347 e-4$ & $3 \cdot 737 e-3$ \\
\hline$-15^{\circ}$ & $2 \cdot 003 e-3$ & $3 \cdot 603 e-6$ & $4 \cdot 538 \mathrm{e}-4$ & $8 \cdot 164 \mathrm{e}-2$ & $2 \cdot 157 \mathrm{e}-3$ & $3 \cdot 947 e-3$ \\
\hline$-30^{\circ}$ & $2 \cdot 202 \mathrm{e}-3$ & $2 \cdot 435 \mathrm{e}-6$ & $8 \cdot 022 \mathrm{e}-4$ & $9 \cdot 988 \mathrm{e}-2$ & $1.559 \mathrm{e}-3$ & $4.524 \mathrm{e}-3$ \\
\hline$-45^{\circ}$ & $2 \cdot 225 \mathrm{e}-3$ & $3 \cdot 811 \mathrm{e}-6$ & $8 \cdot 697 e-4$ & $8 \cdot 781 e-2$ & $6 \cdot 615 e-4$ & $2 \cdot 970 \mathrm{e}-3$ \\
\hline$-60^{\circ}$ & $2 \cdot 141 \mathrm{e}-3$ & $5 \cdot 563 e-6$ & $6 \cdot 930 \mathrm{e}-4$ & $8 \cdot 712 \mathrm{e}-2$ & $3 \cdot 037 \mathrm{e}-4$ & $3 \cdot 334 \mathrm{e}-3$ \\
\hline$-75^{\circ}$ & $2 \cdot 058 \mathrm{e}-3$ & $6 \cdot 862 \mathrm{e}-6$ & $5 \cdot 349 \mathrm{e}-4$ & $9 \cdot 508 \mathrm{e}-2$ & $8 \cdot 562 \mathrm{e}-4$ & $1 \cdot 239 \mathrm{e}-3$ \\
\hline$-90^{\circ}$ & $1.502 \mathrm{e}-3$ & $2 \cdot 965 \mathrm{e}-6$ & $9 \cdot 167 \mathrm{e}-4$ & $8 \cdot 783 e-2$ & $6 \cdot 616 e-4$ & $2 \cdot 974 \mathrm{e}-4$ \\
\hline
\end{tabular}

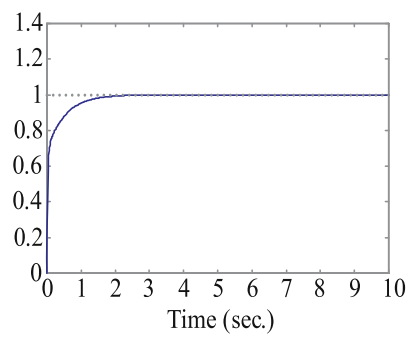

(a)

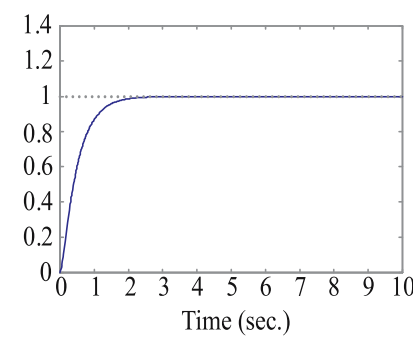

(b)
Figure 5. Step responses of (a) $X$ axis and (b) $Y$ axis driving loops with $\theta=15$ degrees.

\subsection{Case 2: Cross-coupled system with neural compensation}

Following the same setting, the control system with an ANN decoupling compensator is included to eliminate the coupled error. The population for EA is 15. The parameters converge after 25 generations of evolution. The resulting step responses are shown in figure 7 when $\theta$ is 15 degrees. It is easily seen from figures 6 and 7 that performance of the current control system is better than the system without decoupling compensation. See table 2 for the comparison of performance measures.

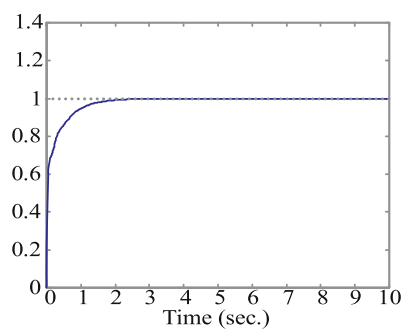

(a)

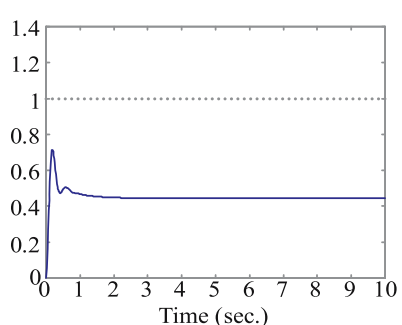

(b)
Figure 6. (a) $X$ axis and (b) $Y$ axis step responses for the cross-coupling system without the ANN compensator while $\theta=15$ degrees. 


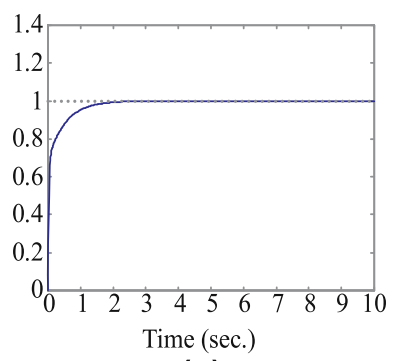

(a)

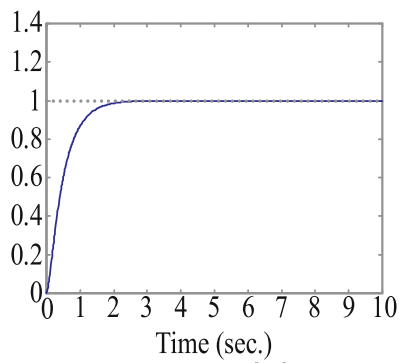

(b)
Figure 7. Step responses of (a) $X$ axis and (b) $Y$ axis driving loops with the ANN decoupling compensator while $\theta=15$ degrees.

Table 2. Performance measures for the system without/with neural decoupling compensation.

\begin{tabular}{lcl}
\hline \multicolumn{3}{c}{$\begin{array}{c}\text { performance measures } \\
\text { angle: } 15 \text { deg. }\end{array}$} \\
\hline Steady-state error-x axis & $1.82 \times 10^{-4}$ & $1.25 \times 10^{-4}$ \\
Steady-state error-y axis & 0.556 & $1.34 \times 10^{-4}$ \\
Max. overshoot-x axis & $1.25 \%$ & $0.017 \%$ \\
Max. overshoot-y axis & $28.5 \%$ & $0.022 \%$ \\
\hline
\end{tabular}

\subsection{Case 3: Curve tracking accuracy}

Tracking accuracy of the cross-coupling system with ANN decoupling compensation for the curve tracking from -90 increasing to 0 degrees is displayed in figure 8 which shows a significant improvement with the maximum contour error reduced to $13 \mu \mathrm{m}$. Figure 9 further

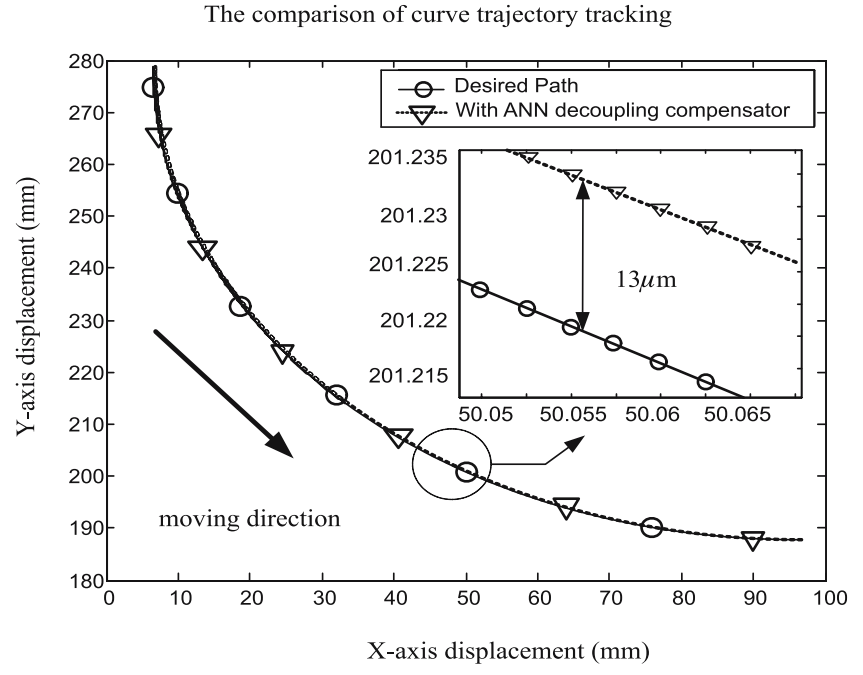

Figure 8. Comparison of curve trajectory tracking with ANN decoupling compensation from -90 to 0 degrees. 


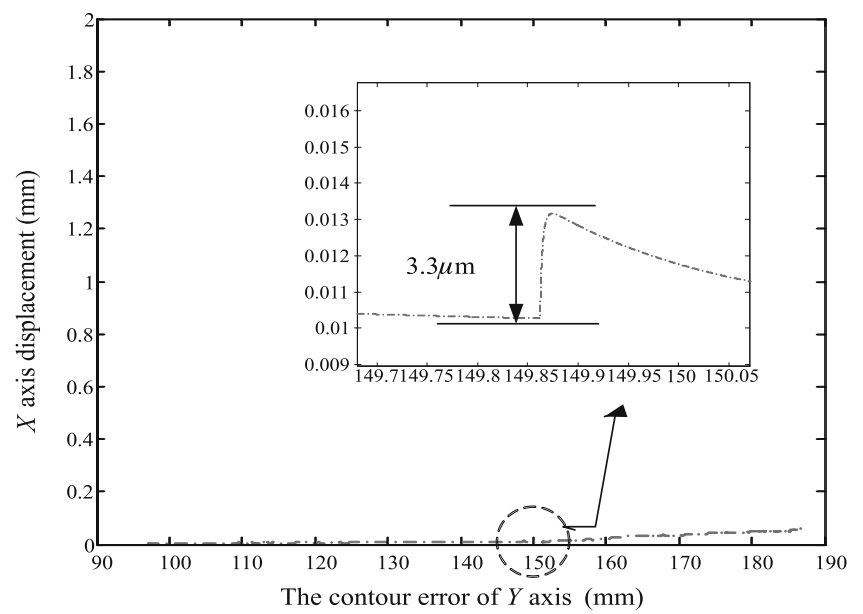

(a)

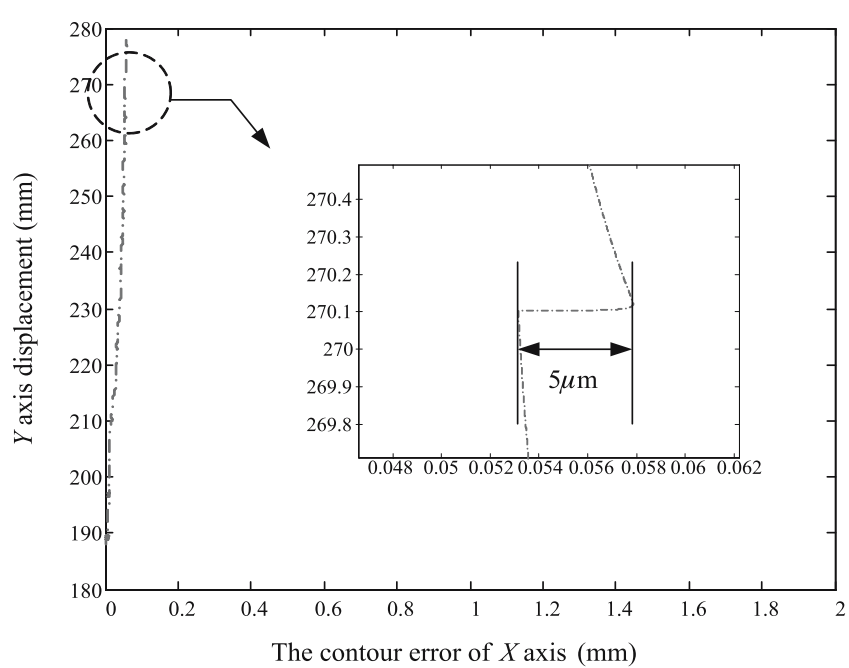

(b)
Figure 9. Contour error of $Y$ axis ( $X$ axis) with ANN decoupling compensator while the moving displacement on $X$ axis ( $Y$ axis) is fixed for the curve tracking from -90 increasing to 0 degrees.

demonstrates precision tracking for one axis while another axis remained fixed. It is observed from these results that the ANN decoupling compensator is very effective to avoid the error growth no matter which axis is considered. The contour error with ANN decoupling compensation is less than $3.3 \mu \mathrm{m}$ and $5 \mu \mathrm{m}$ as the moving displacement on the $X$ and $Y$ axes are fixed respectively. Furthermore, the amplitude of variations in the contour error is refrained as well.

\subsection{Case 4: Application to watch cambered surface polishing system}

The design idea has been experimentally applied to a watch cambered surface polishing system and figure 10 displays the set-up for this surface polishing. The system was required to simultaneously conduct the planar and cambered surface polish which consists of linear motion and rotation; see figure 11 for the illustration of the platform and its working zone. Graphically illustrated in figure 11a is the relationship between each coordinates, especially 


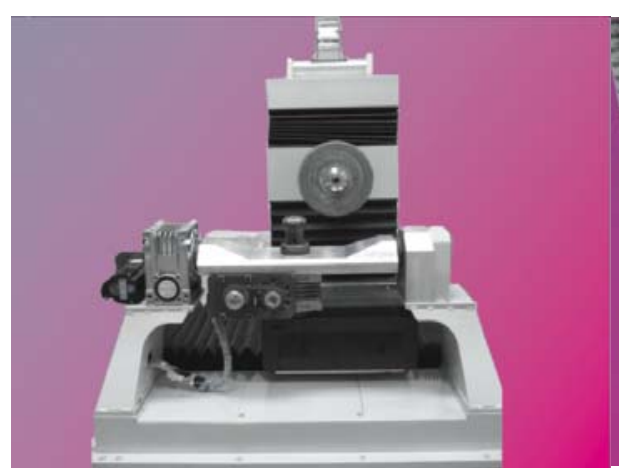

(a)

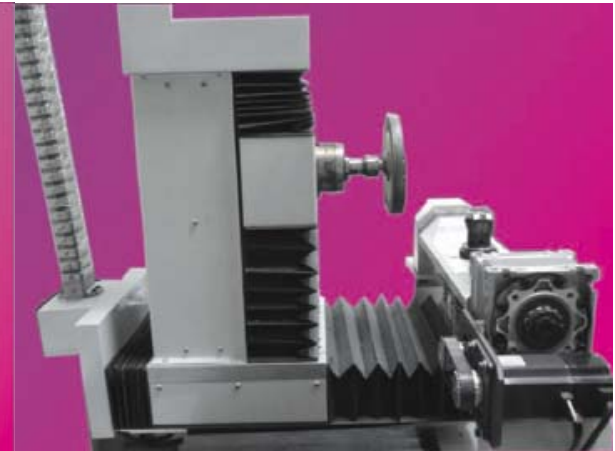

(b)

Figure 10. (a) The front view of watch cambered surface polishing platform; (b) the lateral view of watch cambered surface polishing platform.

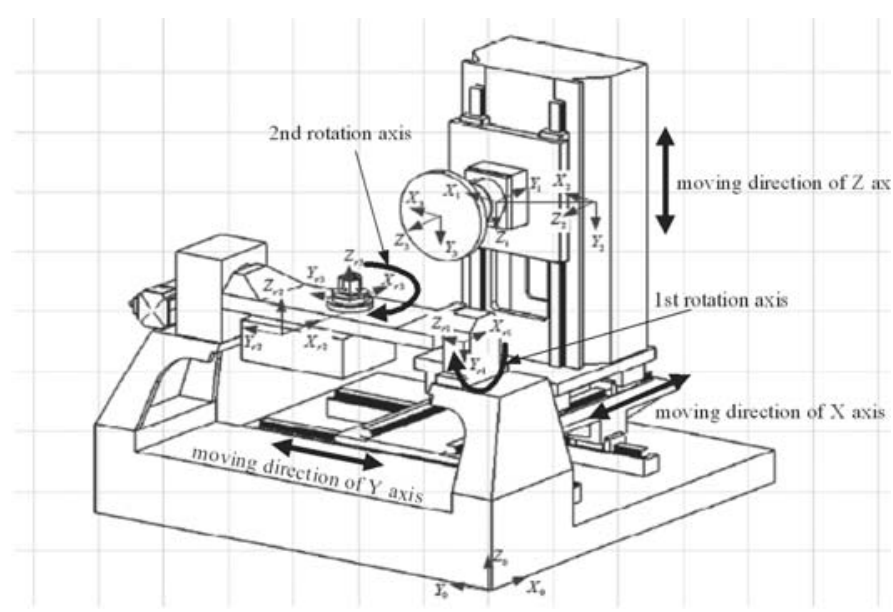

(a)

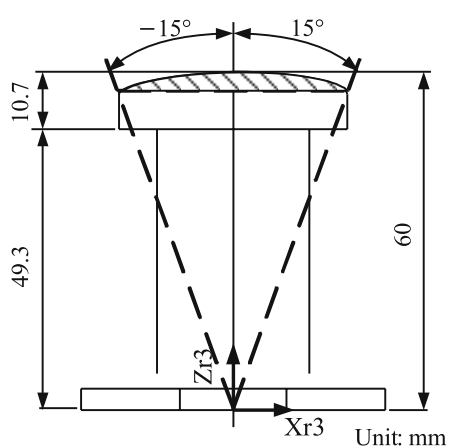

(b)

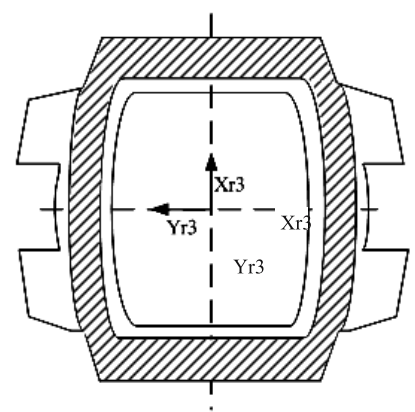

(c)

Figure 11. (a) Coordinate systems of the watch cambered surface polishing platform; (b) the cambered surface polishing area from -15 to 15 degrees; (c) the cambered surface. 
for the platform base $\left(X_{0}, Y_{0}, Z_{0}\right)$ and the watchcase $\left(X_{r 3}, Y_{r 3}, Z_{r 3}\right)$. The system consists of three linear moving axes and two rotating axes where the rotating axes include rotation of an upholder and a clamping apparatus. For convenience of cambered surface polishing, the platform has to move the first touching point through the introduced axes. As the touching point is reached, $Y$ and $Z$ axes are fixed and the angles of the first and second rotation axes are set to be 0 and 90 degrees, respectively. The polishing path is constituted by linear motion along the $X$ axis and rotation around the first rotation $\phi$ axis of the upholder. Following the developed cross-coupling design technique, the reference commands of the $\phi$ and $X$ axes were considered with the range of the working angle $\theta_{\phi}$ from -15 to 15 degrees. The curvature indicated by $\theta_{\phi}$ is the working region on the watch surface to be polished (see figure 11b). The contour error occurs when the simultaneous movement of rotation and linear motion. In other words, if the polishing position of a watch is arrived by the first rotation axis, the linear movement of the $X$ axis has to be arrived at the same time. The geometric relation of the contour error for the current case to the former trajectory tracking problem is $\phi \leftrightarrow X$ and $X \leftrightarrow Y$.

Transfer functions of the $\phi$ and $X$ axis motion systems were, respectively, given by

$$
G_{p \phi}(s)=\frac{266 \cdot 15}{s(s+12 \cdot 571)}(\mathrm{m} / \mathrm{V}), \quad G_{p x}(s)=\frac{171 \cdot 2}{s(s+13 \cdot 82)}(\mathrm{m} / \mathrm{V})
$$

Referring to figures $11 \mathrm{~b}$ and $11 \mathrm{c}$, the coordinate of the watch cambered surface polishing based on $X_{r 3}, Y_{r 3}$, and $Z_{r 3}$ are

$$
\left[\begin{array}{c}
p_{X_{r 3}} \\
p_{Y_{r 3}} \\
p_{Z_{r 3}} \\
1
\end{array}\right]=\left[\begin{array}{c}
60 \sin \theta_{\phi} \\
0 \\
60 \cos \theta_{\phi} \\
1
\end{array}\right] .
$$

The movement along the $X, Y, Z$ axes is characterized by

$$
\begin{gathered}
{\left[\begin{array}{cccc}
c \theta_{r 1} & -c \theta_{r 1} s \theta_{r 2} & s \theta_{r 1} & d_{r 2} s \theta_{r 1}+X_{\text {roffset }} \\
s \theta_{r 2} & c \theta_{r 2} & 0 & d_{r 1}+Y_{\text {roffset }} \\
-s \theta_{r 1} c \theta_{r 2} & s \theta_{r 1} s \theta_{r 2} & c \theta_{r 1} & d_{r 2} c \theta_{r 1}+Z_{\text {roffset }} \\
0 & 0 & 0 & 1
\end{array}\right]\left[\begin{array}{c}
p_{X_{r 3}} \\
p_{Y_{r 3}} \\
p_{Z_{r 3}} \\
1
\end{array}\right]} \\
=\left[\begin{array}{cccc}
0 & 0 & -1 & d_{2}+X_{\text {offset }}-X_{\text {move }} \\
1 & 0 & 0 & Y_{\text {offset }}-Y_{\text {move }} \\
0 & -1 & 0 & Z_{\text {offset }}-Z_{\text {move }} \\
0 & 0 & 0 & 1
\end{array}\right]\left[\begin{array}{c}
p_{X_{3}} \\
p_{Y_{3}} \\
p_{Z_{3}} \\
1
\end{array}\right],
\end{gathered}
$$

where $\left[\begin{array}{llll}p_{X_{3}} & p_{Y_{3}} & p_{Z_{3}} & 1\end{array}\right]^{T}=\left[\begin{array}{llll}30 & 50 & 20 & 1\end{array}\right]^{T}(\mathrm{~mm})$ is the initially contacting position of the polishing wheel. $\theta_{r 1}$ and $\theta_{r 2}$ are the rotating angles for the first and second rotation axes; $s \theta_{r i} \triangleq \sin \theta_{r i}$ and $c \theta_{r i} \triangleq \cos \theta_{r i} ; d_{r 1}$ is the distance of the $X$-axis between the coordinates of the first and second rotation axes; $d_{r 2}$ is the distance between the coordinates of the watchcase 
and the second rotation axes on the $X$-axis; $d_{2}$ is the distance between the coordinates of the polishing wheel and its base on the $X$-axis. The former equation can be rewritten as (unit: $\mathrm{mm}$ )

$$
\begin{aligned}
X_{\text {move }}= & -p_{X_{r 3}} c \theta_{r 1} c \theta_{r 2}+p_{Y_{r 3}} c \theta_{r 1} s \theta_{r 2}-p_{Z_{r 3}} s \theta_{r 1} \\
& -X_{r o f f s e t}-20-d_{2}+X_{o f f s e t}, \\
Y_{\text {move }}= & -p_{X_{r 3}} s \theta_{r 2}-p_{Y_{r 3}} c \theta_{r 2}-d_{r 1}-Y_{\text {roffset }}-30+Y_{\text {offset }}, \\
Z_{\text {move }}= & p_{X_{r 3}} s \theta_{r 1} c \theta_{r 2}-p_{Y_{r 3}} s \theta_{r 1} s \theta_{r 2}-p_{Z_{r 3}} c \theta_{r 1} \\
& -d_{r 2} c \theta_{r 1}-Z_{r o f f s e t}-50+Z_{o f f s e t},
\end{aligned}
$$

with and the following settings:

$$
\begin{aligned}
X_{o f f s e t} & =455, Y_{o f f s e t}=498 \cdot 5, Z_{o f f s e t}=479 \cdot 15, \\
X_{\text {roffset }} & =180, Y_{\text {roffset }}=180, Z_{\text {roffset }}=346 \cdot 15, \\
d_{2} & =158, d_{r 1}=225, d_{r 2}=16 \cdot 5 .
\end{aligned}
$$

PID controller gains were chosen to meet the time domain performance indices, i.e. the rise time should be less than $1.5 \mathrm{sec}$, the maximum overshoot should be less than $5 \%$ and zero steady state error. The characteristic equations for the $\phi$ and $X$ axis control systems are

$$
\begin{aligned}
& \Delta_{\phi}(s)=s^{3}+\left(12 \cdot 571+266 \cdot 15 k_{d}\right) s^{2}+266 \cdot 15 k_{p} s+266 \cdot 15 k_{i} \\
& \Delta_{x}(s)=s^{3}+\left(13 \cdot 82+171 \cdot 2 k_{d}\right) s^{2}+171 \cdot 2 k_{p} s+171 \cdot 2 k_{i}
\end{aligned}
$$

and the following control gains are chosen to meet the required performance specifications:

$$
\begin{aligned}
& k_{d \phi}=0.6741, k_{p \phi}=50.7922, k_{i \phi}=1622.53, \\
& k_{d x}=1.0407, k_{p x}=78.9624, k_{i x}=2522.41 .
\end{aligned}
$$

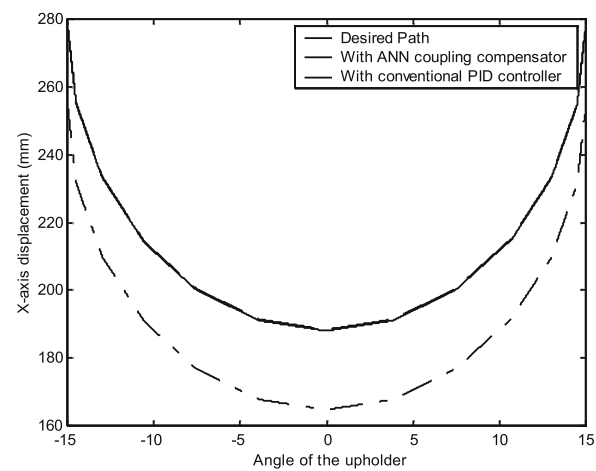

(a)

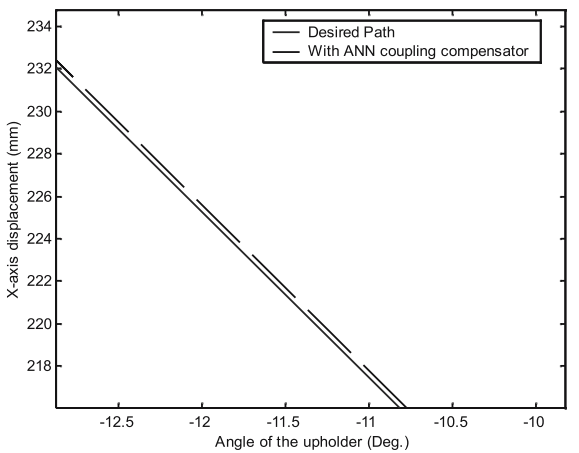

(b)

Figure 12. The cambered surface polishing path; (a) the whole path tracking, (b) local enlargement of the path tracking. 


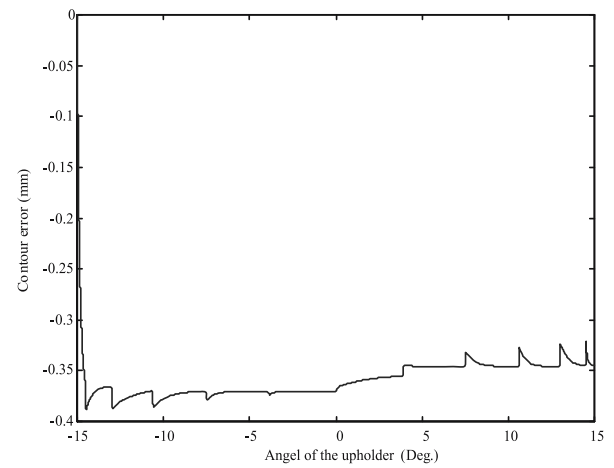

(a)

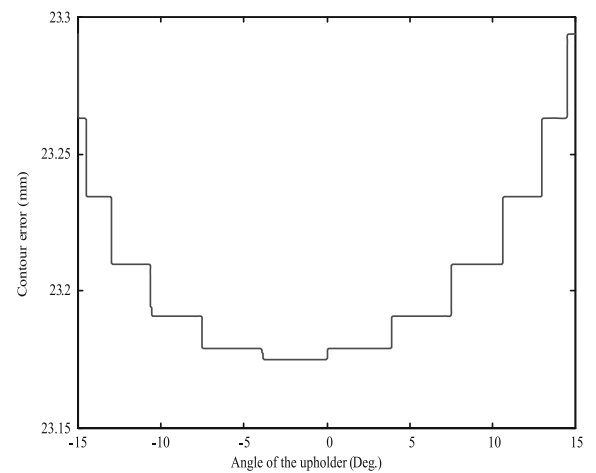

(b)

Figure 13. Contour error with $\theta$ (a) ANN as the decoupling compensator, (b) PID controller as the decoupling compensator.

The same network structure of the ANN in Case 2 was adopted. In addition to the ANN decoupling compensator, a PID controller has also been attempted as a decoupling compensator to reduce the contour error. By the EA with the same fitness function, after 1000 generations of evolution, a set of the optimal PID control gains was obtained as

$$
k_{d c}=0.00214, k_{p c}=0.06293, k_{i c}=0.0001 .
$$

Figure 12 shows the desired and actual trajectories with the angle $\theta$ (the angle of the linear contour with respect to the $\phi$ axis) where the ANN or the PID controller were respectively used as the decoupling compensator. $\theta$ was set from -90 to 90 degrees. Figure 13 shows the resulting contour error. It is easy to perceive excellence of the ANN decoupling compensation scheme.

\section{Conclusions}

A new neural net-based decoupling control scheme for a dual-axis motion platform that integrates two individual PID controllers and a neural network is proposed. The configuration enables synchronous motion of two motor drives which work cooperatively to achieve the desired curve tracking. The compensation scheme significantly reduces the contour error owing to the cross coupling effect. Robust stability conditions are established and applied to the example as a stabilizing control design constraint. An EA involving parallel computation and real-coding strengthens the searching efficiency for control parameters and ANN's connecting weights in the feasible solution space. The experimental results for a watch cambered surface polishing system show that the proposed approach is practical and is capable of dealing with the complicated multiple-axis motion control problem.

This research was sponsored in part by the Ministry of Education, Taiwan, R.O.C. under the ATU plan. 


\section{List of symbols}

$\begin{array}{ll}C_{c} & \text { Decoupling controller } \\ d & \text { Decoupling signal } \\ e_{x}, e_{y} & \text { Tracking errors with respect to the } X \text { and } Y \text { axes } \\ F_{h}, F_{o} & \text { Hidden layer and output layer } \\ k_{p x}, k_{i x}, k_{d x} & \text { PID control gains for the } X \text { axis control subsystem } \\ k_{p y}, k_{i y}, k_{d y} & \text { PID control gains for the } Y \text { axis control subsystem } \\ W & \text { Connecting weight matrix of the neural network } \\ \varepsilon & \text { Contour error } \\ \lambda & \text { Number of offsprings } \\ \theta & \text { Angle of the linear contour with respect to the } X \text { axis } \\ \theta_{\phi} & \text { Working angle of the } \phi \text { axis } \\ \sigma & \text { Standard deviation } \\ \omega & \text { Number of parents } \\ \psi & \text { Activation function. }\end{array}$

\section{References}

Bäck T 1996 Evolutionary algorithms in theory and practice. (New York, USA: Oxford)

Borenstein J, Koren Y 1985 A mobile platform for nursing robots. IEEE Trans. Ind. Electron. 32(2): $158-165$

Choi K J, Lee Y H, Moon J W, Park C K, Harashima F 2007 Development of an automatic stencil inspection system using modified hough transform and fuzzy logic. IEEE Trans. Ind. Electron. 54(1): 604-611

Davis L 1991 Handbook of genetic algorithms. (New York, USA: Van Nostrand Reinhold)

Fang J, Xi Y 1997 Neural network design based on evolutionary programming. Artif. Intell. Eng. 11(2): 155-161

Feng L, Koren Y, Borenstein J 1993 Cross-coupling motion controller for mobile robots. IEEE Control Syst. Mag. 13(6): 35-43

Han W, Jafari M A 2007 Coordination control of positioning and deposition in layered manufacturing. IEEE Trans. Ind. Electron. 54(1): 651-659

Hashimoto H, Kubota T, Sato M, Harashima F 1992 Visual control of robotic manipulator based on neural networks. IEEE Trans. Ind. Electron. 39(6): 490-503

Inshguro A, Furuhashi T, Okumura S 1992 A neural network compensator for uncertainties of robotics manipulator. IEEE Trans. Ind. Electron. 39(6): 565-570

Kim S H, Park C, Harashima F 2001 A self-organized fuzzy controller for wheeled mobile robot using an evolutionary algorithm. IEEE Trans. Ind. Electron. 48(6): 464-474

Kim M K, Lee C G, Jung, H K, 1998 Multiobjective optimal design of three-phase induction motor using improved evolution strategy. IEEE Trans. Magn. 34(5): 2980-02983

Kuo S K, Shan X, Menq C H 2003 Large travel ultra precision $x-y-\theta$ motion control of a magneticsuspension Stage. IEEE-ASME Trans. Mechatron. 8(3): 334-341

Liu Z Z, Luo F L, Azizur R M 2005 Robust and precision motion control system of linear-motor direct drive for high-speed X-Y table positioning mechanism. IEEE Trans. Ind. Electron. 52(5): $1357-1363$

Moallem M, Mirzaeian B, Mohammed O A, Lucas C, 2001 Multi-objective genetic-fuzzy optimal design of PI controller in the indirect field oriented control of an induction motor. IEEE Trans. Magn. 37(5): 3608-3612

Park E C, Lim H, Choi C H 2003 Position control of X-Y table at velocity reversal Using presliding friction characteristics. IEEE Trans. Control Syst. Technol. 11(1): 24-31 
Subbu R, Goebel K, Frederick D K 2005 Evolutionary design and optimization of aircraft engine controllers. IEEE Trans. Syst. Man Cybern. Part C-Appl. Rev. 35(4): 554-565

Wai R J, Tu C H 2007 Design of total sliding-mode-based genetic algorithm control for hybrid resonant-driven linear piezoelectric ceramic Motor. IEEE Trans. Power Electron. 22(2): 563-575 Zhou K, Doyle J C 1998 Essentials of robust control. (New Jersey, USA: Prentice-Hall) 